\section{Review: Screening and intervention for alcohol misuse in emergency rooms reduces alcohol-related injuries}

\section{QUESTION}

Question: What is the evidence for emergency room based interventions for alcohol use?

Outcomes: Frequency or quantity of alcohol consumption, frequency of heavy drinking consumption, and consequences during follow-up (for example, alcohol-related injuries).

\section{METHODS}

Design: Systematic review with meta-analysis.

Data sources: MEDLINE, EMBASE, PsychINFO, CCTR, DARE, CDSR, ACP Journal Club, Global Health, SWAB, Current Contents Connect and Web of Science, were searched between January 1996 and July 2007.

Study selection and analysis: Intervention studies published in a peer-reviewed journal examining the effectiveness of emergency room treatment (including counselling, motivational interviewing, pharmacological treatment or advice) on an alcohol-related outcome. The Centers for Disease Control and Prevention Guide to Community Preventative Services Data Collection Instrument criteria and the Cochrane Effective Practise and Organisation of Care Review Group Data Collection Checklist were used to appraise included studies and aid data collection. Only RCTs were pooled in meta-analyses. Standardised mean difference was calculated for continuous outcomes (frequency of alcohol consumption or binge drinking, and drinking consequences) and results pooled using a fixed effects modal. Binary outcomes of alcohol related injuries were reported for two studies and a conversion used for one study reporting them as a continuous outcome to give an algebraic equivalent of an odds ratio. Heterogeneity was investigated using the Cochrane $\chi^{2}$ statistic, with $p<0.10$ indicating heterogeneity.

\section{MAIN RESULTS}

Thirteen intervention studies met the inclusion criteria (10 RCTs, 2 cohort studies and 1 non-randomised controlled trial). Average number of participants in trials was 582. Ten of the trials evaluated one session of counselling (duration
5-60 minutes; 8 of which included motivational interviewing); one evaluated a laptop-based interactive programme (including increasing alcohol knowledge and decreasing intention to misuse); one evaluated personalised computerprinted information (comparing a person's drinking habits to the norm and offering advice and details of treatment available); and one, generic patient information handouts on sensible drinking. The control groups used in the trials varied and mostly included standard care and generic or personalised advice (written or verbal) either singly or combined. Outcomes assessed varied between studies and included: quantity/frequency of alcohol consumption (5 trials); frequency of heavy drinking (7 trials); negative drinking consequences (6 trials); and alcohol-related injuries (3 trials). Few studies reported effect sizes or enough data to calculate effect sizes. Emergency room-based interventions decreased the likelihood of having an alcohol-related injury in the following 6-12 months, compared to control (three RCTs; OR 0.59, CI not reported, $p<0.005 ; \chi^{2}=3.42, p=0.18$ ). Emergency room based interventions did not significantly decrease the frequency of drinking at 12 months (two RCTs; $\mathrm{SMD}-0.14$ ); frequency of heavy drinking at 3 months (three RCTs; SMD 0.03); frequency of heavy drinking at 12 months (five RCTs; SMD 0.03); or drinking consequences at 612 months (four RCTs; SMD -0.14; p value not significant for all listed effect sizes).

\section{CONCLUSIONS}

Brief emergency room based interventions reduce alcohol related injuries, but evidence about their effects on frequency of drinking, heavy drinking or drinking consequences were inconclusive.

\section{ABSTRACTED FROM}

Havard A, Shakeshaft A, Sanson-Fisher R. Systematic review and meta-analyses of strategies targeting alcohol problems in emergency departments: interventions reduce alcohol-related injuries. Addiction 2008;103:368-76.

Correspondence to: Alys Havard, National Drug and Alcohol Research Centre, University of New South Wales, Sydney, NSW 2052, Australia; a.havard@ unsw.edu.au

Source of funding: Alcohol Education and Rehabilitation Foundation of Australia.

- Additional notes and references are published online only at http://ebmh.bmj.com/ content/vol11/issue3 